\section{Psychoedukation: effektiv bei ADHS}

Ein Team von Wissenschaftlern aus Spanien beurteilte die Wirkung der Psychoedukation bei Kindern mit ADHS. Sie benutzen dafür eine systemische Literaturübersicht der Studien veröffentlicht zwischen Januar 1980 und Juli 2010 und kamen zu einem positiven Ergebnis. sychoedukation wird definiert als eine professionell durchgeführte Therapie, die psychotherapeutische und pädagogische Elemente integriert. Dies umfasst Information von Eltern, den Betroffenen und Erziehern über das Störungsbild und über die Kompensationsmöglichkeiten. Um die Effektivität der Psychoeduktion zu bewerten, nahm ein Team von Wissenschaftlern aus Spanien sieben Studien zu diesem Thema mit 2.034 Studienteilnehmern in ihre Bewertung mit auf. Alle sieben Studien kamen zu dem Schluss, dass Psychoedukation einen positiven Einfluss auf die ADHS-Symptomatik hat, die Eltern und die betroffenen Kinder zufriedener seien und Konfliktsituationen weniger häufig vorkämen.

Eine kürzlich erschienene Studie aus Schweden stellte ebenfalls einen additiven Effekt durch Kombination der Psychoedukation mit einer medikamentösen Therapie fest [Svanborg P et al. Eur Child Adolesc Psychiatry 2009; 18: 240-9].

\section{Depressive Väter schlagen häufiger zu}

Aufgrund einer früheren Metaanalyse wird vermutet, dass rund $10 \%$ der Väter innerhalb des ersten Jahres nach der Geburt ihres Kindes eine Depression haben. Eine Studie aus Michigan untersucht die Auswirkungen einer väterlichen Depression auf die Interaktion mit ihren Kindern.
D urch die veränderten gesetzgeberischen Rahmenbedingungen steigt auch in Deutschland die Zahl der Väter, die Elternzeit in Anspruch nehmen. Während Auswirkungen mütterlicher psychischer Erkrankungen auf die Kinder gut dokumentiert sind, gibt es relativ wenig Daten zu den Vätern. In einer Studie aus den USA wurden 1.746 Väter von ein Jahr alten Kindern interviewt. Das Verhalten und die Interaktion der Väter und Kinder wurde anhand der Interviews registriert

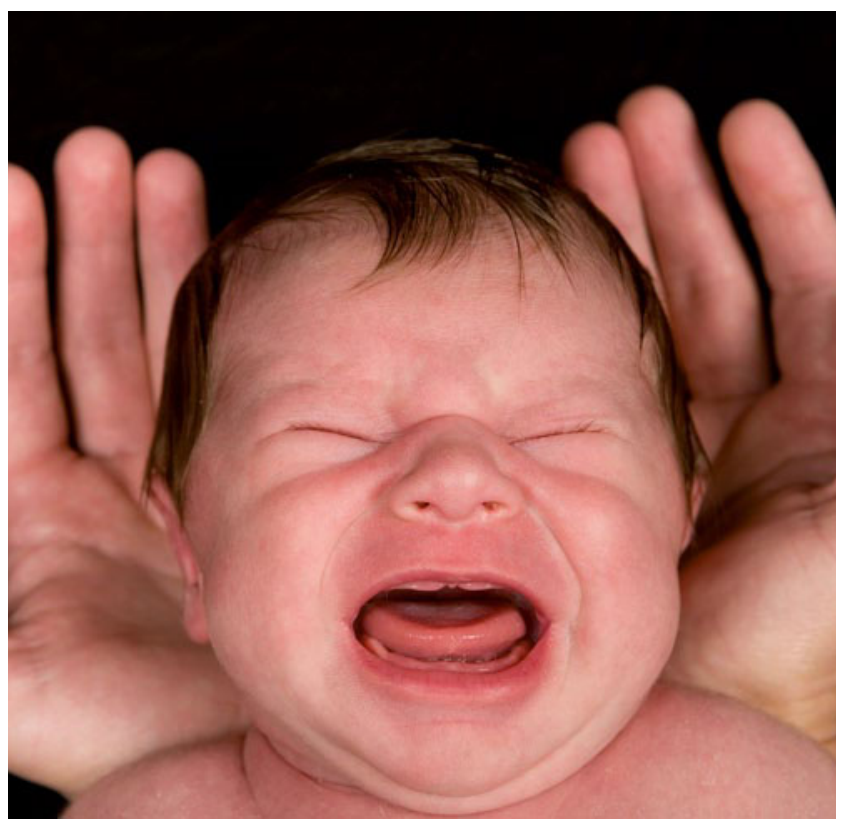

Grund zu weinen: $41 \%$ der Väter mit Depressionen schlagen ihr Kind.
Montoya $A$ et al. Is psychoeducation for parents and teachers of children and adolescents with ADHD efficacious? A systematic literature review. Eur Psychiatry 2011; 26: 166-75

Kommentar: Im Rahmen der multimodalen Therapie wird entsprechend den Leitlinien ADHS der Kinder- und Jugendärzte Psychoedukation als erster therapeutischer Schritt empfohlen. Diese aus dem praktischen medizinischen Alltag entstandene Empfehlung findet jetzt in den Ergebnissen dieser Metaanalyse seine Bestätigung. Die Psychoedukation stellt die Basis für alle weiteren Therapiemaßnahmen dar und verbessert die Compliance für die folgenden therapeutischen Schritte.

Dr. Kirsten Stollhoff und Depressionen mithilfe eines Fragebogens diagnostiziert.

Insgesamt hatten $7 \%$ der Väter eine Depression. Väter mit Depressionen hatten während des letzten Monats vor dem ersten Geburtstag des Kindes signifikant häufiger zugeschlagen ( $41 \%$ gegenüber $13 \%$ der nicht-depressiven Väter). Dies ergibt eine Odds Ratio von 3,92. Zudem hatten depressive Väter ihren Kindern deutlich seltener vorgelesen.

Davis RN et al. Fathers' depression related to positive and negative parenting behaviors with 1-year-old children. Pediatrics 2011; $127: 612-8$

Kommentar: Depressionen bei Vätern, die ihre Kinder erziehen, sind bisher nicht im Fokus des Interesses - auch nicht bei Kinderärzten. Die vorliegende Studie zeigt, dass - wie zu erwarten - auch bei den Vätern Depressionen zu einer veränderten Interaktion mit den Kindern führen. Die Rolle des niedergelassenen Pädiaters liegt in einer Früherkennung einer solchen Störung und in einer entsprechenden Beratung. Interessanterweise hatten $77 \%$ der Väter mit Depression innerhalb des vorangegangenen Jahres Kontakt zum Kinderarzt gehabt und wären damit für eine Beratung oder ein Screening erreichbar. Fazit: Wir alle müssen auch auf die psychische Situation von Vätern eingehen!

Dr. Martin Claßen 